\title{
Reinforced Sleeve versus Non-Reinforced Sleeve Gastrectomy in Morbid Obese Patients: A comparative Study
}

\author{
Mohamed G. Fouly, MD; Ahmed Y. Elrifaie, MD; Ahmed M. Farrag, MD \\ Department of Surgery, Faculty of Medicine, Ain Shams University, Egypt
}

\begin{abstract}
Background: Staple-line leaks after laparoscopic sleeve gastrectomy (LSG) remains a concerning complication in addition to other complications which may occur as bleeding and twisting. Buttressing of the staple line after sleeve gastrectomy is an acceptable reinforcement method but data recorded regarding leaks have been equivocal. Intraoperative measures are used as over-sewing of stapling line to prevent these events and several other methods are used to decrease and control these complications and decrease these burden. Although staple-line reinforcement in several studies is reported to decrease postoperative leakage and bleeding, other studies reported that reinforcement has no role. Authors also reported using buttressing materials. Our study is to compare between sleeve gastrectomy vs. reinforced sleeve gastrectomy with over-sewing of the staple line and omentopexy in morbid obese patients as regards overall complications as bleeding, Leakage and twist.
\end{abstract}

Patients and methods: This prospective randomized Controlled trial involved 500 obese patients conducted during period from January 2018 to January 2019 with follow up till June 2019. The patients were divided into 2 groups, Group A (250 patients) underwent sleeve gastrectomy then reinforcement by suturing of sleeved stomach along the whole length of staple line with omentopexy and Group B (250 patients) underwent sleeve gastrectomy with no over sewing or omental patching.

Results: There was a significant difference between the two study groups as regard operative time with longer mean operative time in omentopexy group. However, no statistically significant difference was found between the two study groups as regard post-operative leakage, hemorrhage and twisting although overall complications was less in reinforcement group and leaks detected in reinforcement group are mostly contained leaks but this was statistically insignificant.

Conclusion: Minimal advantage is added to sleeve gastrectomy with reinforcement by over-sewing and omentopexy as regard post-operative leakage, hemorrhage and twisting although overall complications was less in reinforcement group and leaks detected in reinforcement group are mostly contained leaks but this was statistically insignificant so further studies is needed with larger sample size.

Key words: Reinforced sleeve, twist, leakage, bleeding, omentopexy.

\section{Introduction}

Obesity is a burden on individuals and society. Obesity has rates of death driven by co-morbidities such as hypertension, diabetes, sleep apnea, steatohepatitis, gastro-esophageal reflux disease (GERD), arthritis, polycystic ovary disease and infertility. ${ }^{1-4}$

Sleeve gastrectomy (LSG) was presented as a part of the duodenal switch with bilio-pancreatic diversion. Now, it is universally known primary procedure. ${ }^{5,6}$

LSG is efficient, safe and simple procedure for morbid obesity with improvement in comorbidities and satisfactory results in follow up results. ${ }^{7-9}$
Recent studies demonstrated that LSG is not only a restrictive procedure, as gut hormones interactions e.g. (Ghrelin, PYY, and incretins) were demonstrated. So, hormonal interactions were adding power to action of Sleeve gastrectomy increasing its efficacy and maintenance in loss of weight in follow up data. ${ }^{10-12}$

Leak due to staple-line disruption, bleeding, strictures and twist are reported complications with varying severity and varying methods of management from conservative measures to more interventions, e.g., blood transfusion in bleeding, second-look in more complicated cases, and further interventions as considering conversion to bypass in some cases of 
leak. Leak is the most serious complication and its incidence is reported to be up to $2-5 \% .{ }^{10,13}$

Bleeding after LSG incidence is reported to be $1-6 \%$. Either intraluminal or abdominal bleeding was reported; intra-abdominal bleeding source could be the staple line which in most cases can be conservatively managed. However, second-look laparoscopy for patients with persistent tachycardia (>120 \minute) could be an important option for management. Also second look is indicated when there is continuous drop in hemoglobin level. ${ }^{13-15}$

Intraoperative measures are used as over-sewing of stapling line to prevent these events and several other methods are used to decrease and control these complications and decrease these burden. Staple-line reinforcement is reported to decrease postoperative leakage and bleeding. Authors also reported using buttressing materials such as glycolide-trimethylene carbonate co-polymer to decrease these complications. ${ }^{16-19}$

\section{Aim/ Objectives}

The aim of our study is to compare between Sleeve gastrectomy and Reinforced sleeve gastrectomy with over-sewing of the staple line and omentopexy in morbid obese patients regarding overall complications as bleeding, Leakage and twisting.

\section{Patients and Methods}

This prospective randomized Controlled trial involved 500 obese patients conducted at Ain Shams University Hospitals, El-Hurria Hospital, El-Thuria Hospital during period from January 2018 to January 2019 with follow up till June 2019. The patients were divided into 2 groups, Group A (250 patients) underwent sleeve gastrectomy then reinforcement by suturing of sleeved stomach along the whole length of staple line with omentopexy by PDS 2/0 guided by bougie and Group B (250 patients) underwent Sleeve gastrectomy with no over sewing or omental patching. Ethical approval was taken from Ain Shams University ethical committee and written consent was taken from every patient after explanation of all details of the operation, advantages, disadvantages, realistic expectations and all the possible intra-operative, early and late post-operative complications. Surgeries were done by the same surgical team throughout the study.

\section{Inclusion criteria:}

We included obese male or female patients aging from 18 to 60 years with BMI more than 35 or more than 30 with comorbidities.

\section{Exclusion criteria:}

We excluded from the study patients with previous bariatric surgeries, when laparoscopic sleeve is converted to open sleeve, pregnant or lactating women and patients with psychiatric disorders.

\section{Technique:}

We standardize the procedure in each group. Operative technique of Laparoscopic sleeve gastrectomy: The patient was positioned in French position. Pneumoperitoneum was established at 11 or $14 \mathrm{mmHg}$ using Visiport. Other ports were placed under direct visualization as required to establish feasible and comfortable ergonomics.

(Figures 1,2).

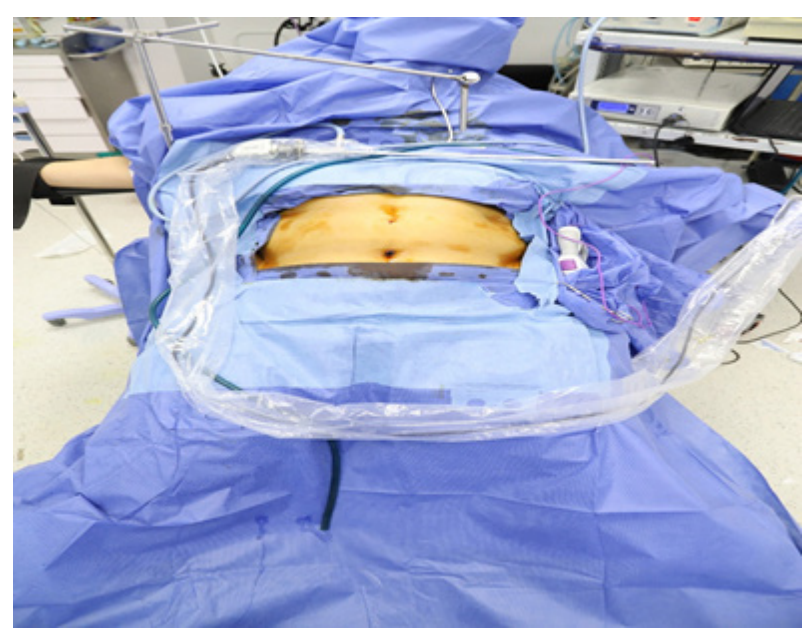

Fig 1: Preparation, draping and positioning after anesthesia.

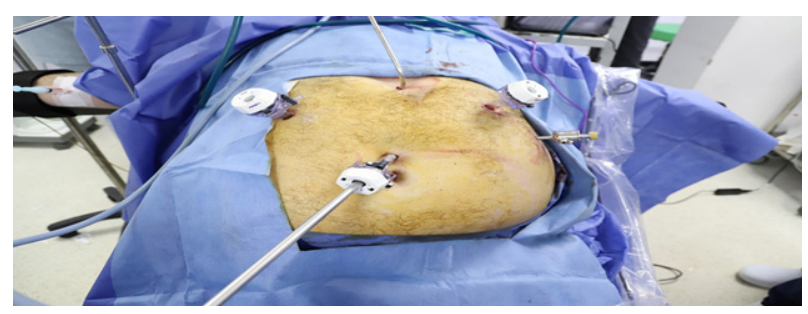

Fig 2: Pneumoperitoneium and ports inserted with a liver retractor.

The stomach was vertically sleeved, guided by 36 Fr bougae. Selecting appropriate staple height to accommodate tissue thickness, slowly stapling to ensure that there was no bleeding; avoiding narrowing near the angularis incisura was ensured.

(Figures 3,4). 


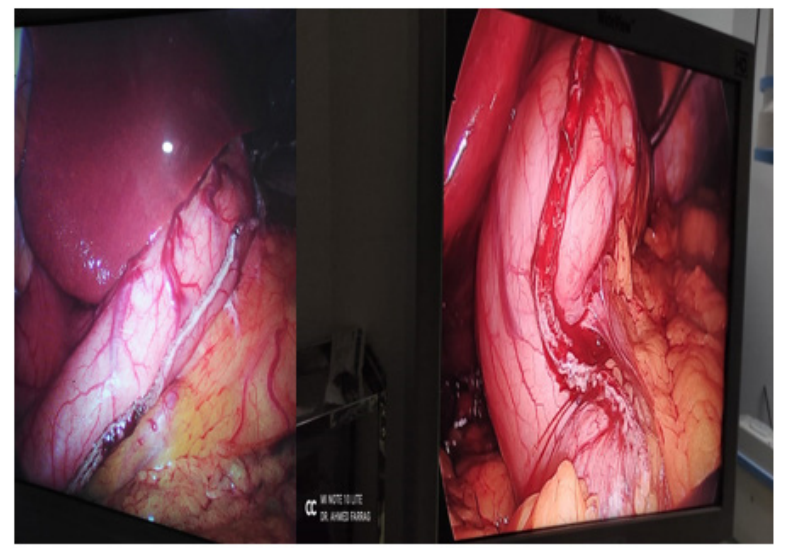

Fig 3: Sleeved stomach without omentopexy before and during methylene blue test.
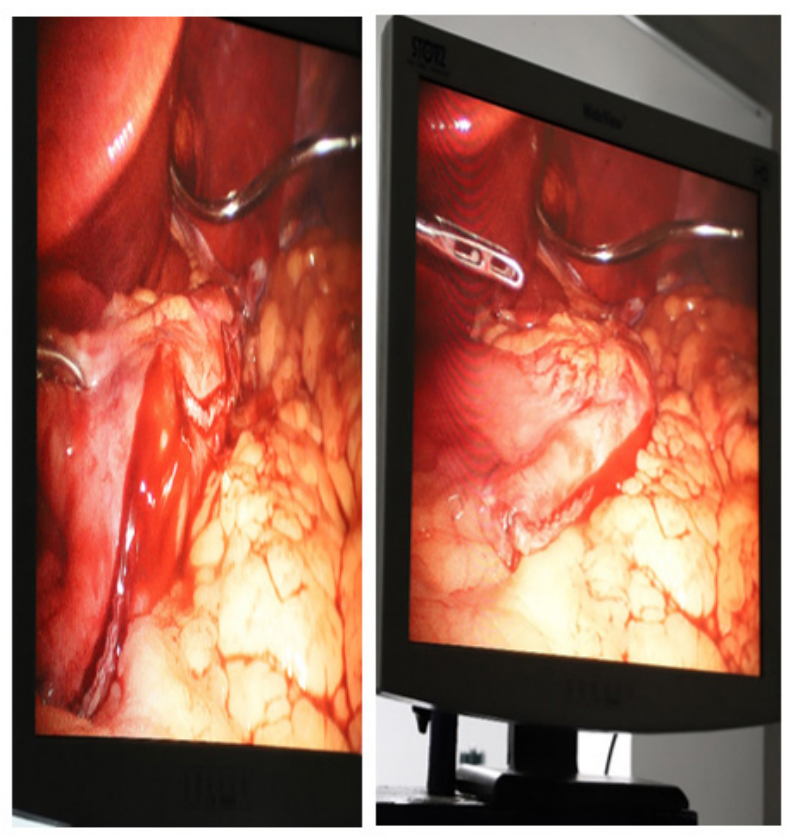

Fig 4: Sleeved stomach after stapling before omentopexy.

In group A suturing of sleeved stomach along the whole length of staple line by PDS $2 / 0$ guided by bougie using an intra-corporeal technique with small bite technique each stitch half $\mathrm{cm}$ apart just beneath the staple line guided by the bougae to avoid stricture was done (Figures 5,6).

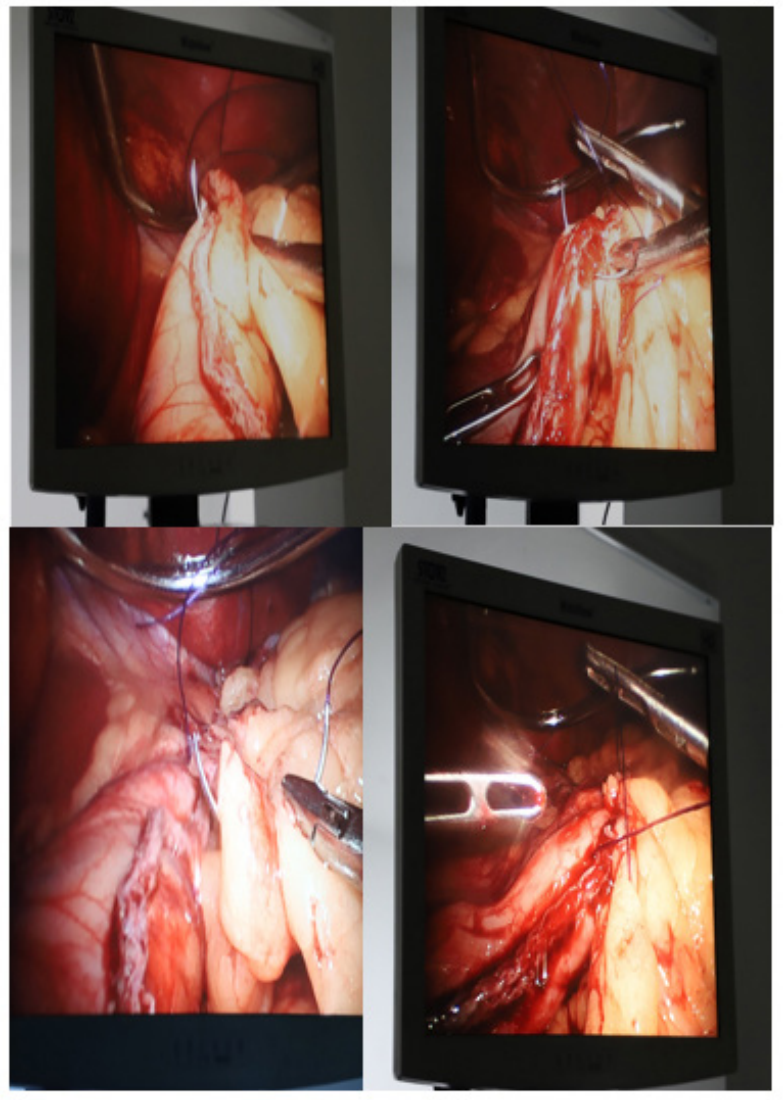

Fig 5: Steps of over-sewing and omentopexy after sleeve gastrectomy.

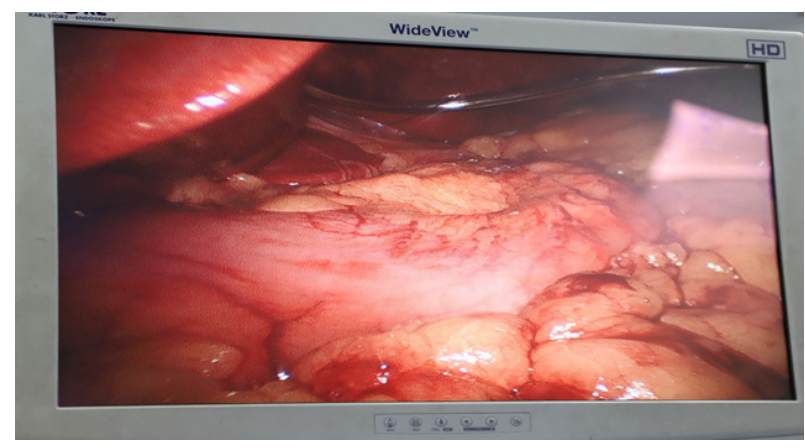

Fig 6: Sleeved stomach after stapling after suture reinforcement with omentopexy.

\section{Follow up of cases:}

Follow up period was at least 6 months. Ultrasound and $\mathrm{CBC}$ (to exclude bleeding and leakage) were done and repeated if needed. Upper GI endoscopy was done for persistent vomiting to exclude twisting or stricture and regular follow up visits were planed after discharge for early detection of any complications. 


\section{Results}

When we compare both groups as regards age there was no significant difference between the two study groups (Table 1) (Figure 7).

Table 1: Description and comparison between the two study groups as regard age

\begin{tabular}{|c|c|c|c|c|c|c|}
\hline & \multicolumn{4}{|c|}{ Group } & \multirow{3}{*}{$\mathbf{P}$} & \multirow{3}{*}{ Sig } \\
\hline & \multicolumn{2}{|c|}{ Without Omentopexy } & \multicolumn{2}{|c|}{ With Omentopexy } & & \\
\hline & Mean & 土SD & Mean & \pm ISD & & \\
\hline Age & 35.79 & 12.59 & 35.58 & 11.81 & 0.849 & NS \\
\hline
\end{tabular}

*Student t test.

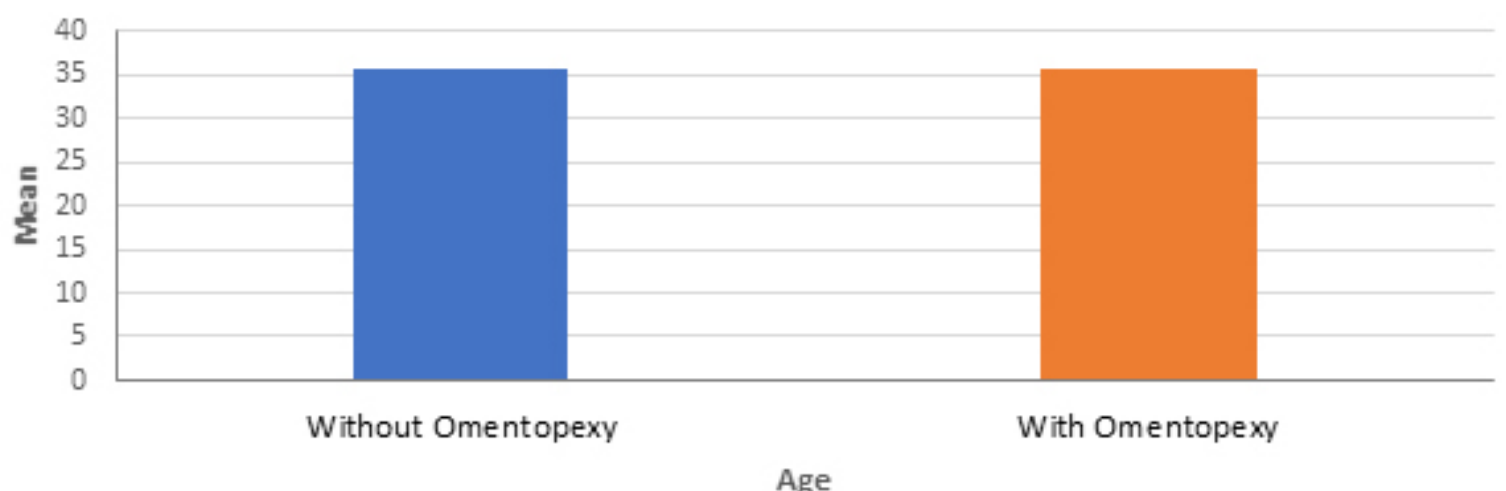

Fig 7: Chart showing the comparison between the two study groups as regard age.

There was a statistically significant difference between the two study groups as regard Operative time with longer mean operative time in omentopexy group (77.1 min vs. 48.2 min). However, no statistically significant difference was found between the two study groups as regard post-operative leakage, hemorrhage and twisting although complicated cases were less in reinforcement groups and leaks detected after reinforcement are mostly contained leaks (Tables 2,3) \& (Figure 8).

Table 2: Description and comparison between the two study groups as regard operative and post-operative data (operative time, leakage)

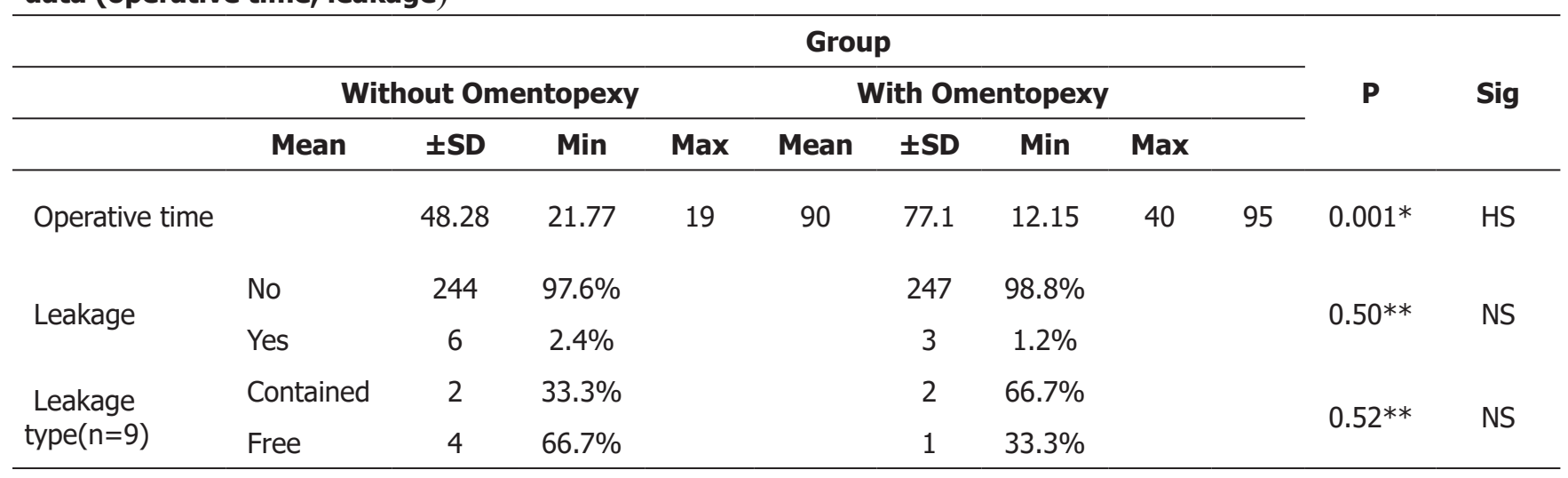

* Student t test.

** Fisher's Exact Test. 
Table 3: Description and comparison between the two study groups as regard operative and post-operative data (bleeding, twist)

\begin{tabular}{|c|c|c|c|c|c|c|c|}
\hline & \multicolumn{5}{|c|}{ Group } & \multirow{3}{*}{$\mathbf{P}$} & \multirow{3}{*}{ Sig } \\
\hline & \multicolumn{2}{|c|}{ Without Omentopexy } & \multicolumn{2}{|c|}{ With Omentopexy } & & & \\
\hline & Mean & $\pm S D$ & Mean & $\pm S D$ & & & \\
\hline \multirow{2}{*}{ Hemorrhage } & No & 248 & $99.2 \%$ & 249 & $99.6 \%$ & \multirow{2}{*}{$1.0 * *$} & \multirow{2}{*}{ NS } \\
\hline & Yes & 2 & $0.8 \%$ & 1 & $0.4 \%$ & & \\
\hline \multirow{2}{*}{ Twisting } & No & 248 & $99.2 \%$ & 249 & $99.6 \%$ & \multirow{2}{*}{$1.0 * *$} & \multirow{2}{*}{ NS } \\
\hline & Yes & 2 & $0.8 \%$ & 1 & $0.4 \%$ & & \\
\hline
\end{tabular}

*Student t test.

**Fisher's Exact Test.

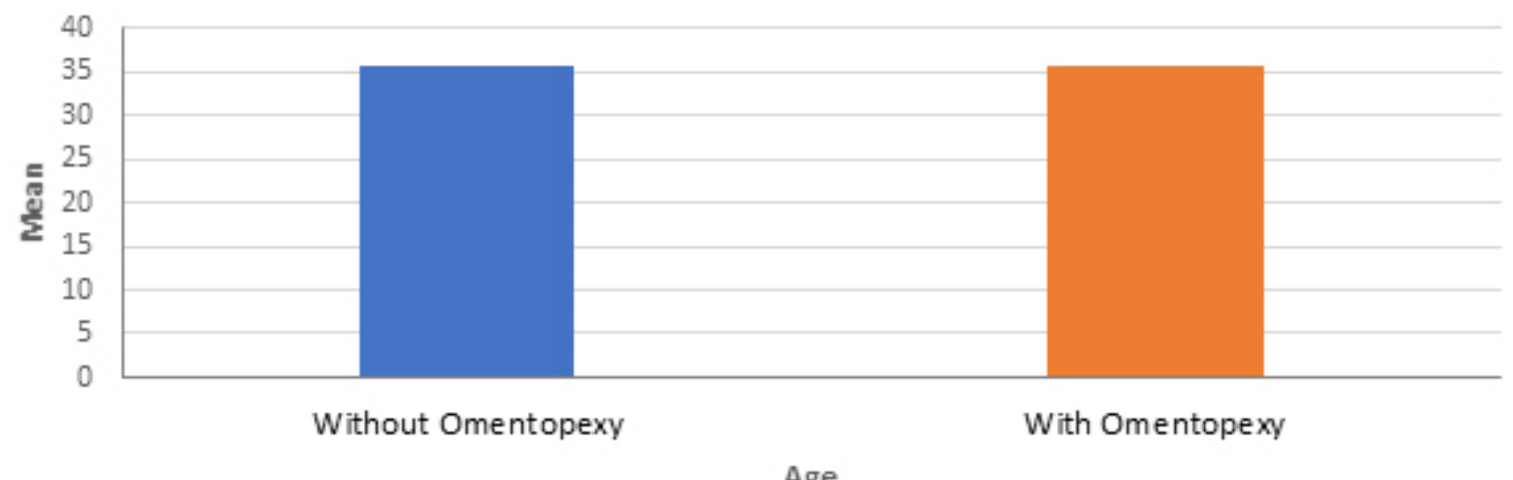

Age

Fig 8: Chart showing the comparison between the two study groups as regard operative time.

\section{Discussion}

By revision of the literature we found leak rate vary between 1 and 3\%, and overall mortality correlated to leak is about $9 \% .{ }^{20-22}$ Our study is conducted on 500 patients showed that there was no significant difference between the two study groups as regard age. So, age could not be a confounder in our study between both groups. Our comparative prospective study found that omentopexy is a safe efficient technique, with satisfactory results on bleeding, leaks and twist.

Overall complications were less in reinforcement group. There was a statistically highly significant difference between the two study groups as regard Operative time with longer mean operative time in reinforcement group (77.1 min vs. $48.2 \mathrm{~min}$ ). A recent randomized study which compares invagination of stable line after suturing to no reinforcement demonstrated that leak rates is reduced for the suturing with invagination group, although this costs a higher operative time by average of 18 $\min .{ }^{23}$ Increased cost and operative time with intracorporeal suturing is also demonstrated in other studies that reported an extra 13 to 24 min per case. $^{24,25}$

However, in no reinforcement group (250 patients); leaks occurred in 6 patients $(2.4 \%) 2$ of them are contained, bleeding occurred in two cases and twisting in two cases. In reinforcement group (250 patients); leaks occurred in 3 patients (1.2\%) 2 of them are contained, one case had bleeding and other one had twist. Thus, no statistically significant difference was found between the two study groups as regard post-operative leakage, hemorrhage and twist.

Twisting may be presented early by salivation and repeated episodes of vomiting. Late may be presented with repeated vomiting of food which may be undigested and reflux which could be refractory to medications. ${ }^{26}$ Twisting of the sleeved stomach may be due to improper alignment of stapling during firing which may be caused by unequal traction on the greater curvature of the stomach which may lead to twist of gastric tube either posteriorly or anteriorly. ${ }^{27}$

Upper GI endoscopy is an effective tool in diagnosis 
of a twist. ${ }^{28}$ Although difference in twist detected in both groups was statistically insignificant, less twist in reinforcement group was noticed in our study with only one case in reinforcement group presented with persistent vomiting and twist was diagnosed with upper gastrointestinal endoscopy and the patient improved conservatively in contrast to two cases of twist in no reinforcement group detected with upper endoscopy after persistent vomiting presentation one of them conservatively improved and the other was converted to one anastomosis gastric bypass.

It is also supported with a study that found that reattachment of the omentum with sutures to the gastric tube after stapling is effective to prevent gastric twisting. ${ }^{29}$

Although overall complications was less in reinforcement group and leaks detected in reinforcement group are mostly contained leaks but this was statistically insignificant so further studies is needed with larger sample size.

This is supported with a large randomized prospective trial that showed that there was no significant difference between reinforcement and without reinforcement after sleeve gastrectomy, in terms of leakage rate. ${ }^{19}$

Authors also demonstrated that bleeding rate was decreased in reinforcement group and confirmed that buttressing after laparoscopic sleeve gastrectomy did not determine statistical significant evidence on leaks. ${ }^{30}$

\section{Conclusion}

Minimal advantage is added to sleeve gastrectomy with reinforcement by over-sewing and omentopexy as regard post-operative leakage, hemorrhage and twisting although overall complications was less in reinforcement group and leaks detected in reinforcement group are mostly contained leaks but this was statistically insignificant so further studies is needed with larger sample size.

\section{References}

1. Arroyo-Johnson C, Mincey KD: Obesity epidemiology worldwide. Gastroenterol Clin N Am. 2016; 45(4): 571-9.

2. D. Fuks, P. Verhaeghe, O. Brehant, C. Sabbagh, F. Dumont, M. Riboulot, et al: Regimbeau, Results of laparoscopic sleeve gastrectomy: A prospective study in 135 patients with morbid obesity, Surgery. 2009; 145(1): 106-113.

3. Haslam DW, James WP: Obesity. Lancet (London, England). 2005; 366(9492): 1197-209.
4. Abdelaal M, le Roux CW, Docherty NG: Morbidity and mortality associated with obesity. Ann Transl Med. 2017; 5(7): 161.

5. Lalor PF, Tucker ON, Szomstein $\mathrm{S}$, et al: Complications after laparoscopic sleeve gastrectomy. Surg Obes Relat Dis. 2008; 4(1): 33-8.

6. Mechanick JI, Youdim A, Jones DB, et al: Clinical practice guidelines for the perioperative nutritional, metabolic, and nonsurgical support of the bariatric surgery patient-2013 update: cosponsored by American Association of Clinical Endocrinologists, The Obesity Society, and American Society for Metabolic \& Bariatric Surgery. Obesity (Silver Spring, Md). 2013; 21(Suppl 1): S1-27.

7. Menenakos E, Stamou KM, Albanopoulos $\mathrm{K}$, et al: Laparoscopic sleeve gastrectomy performed with intent to treat morbid obesity: A prospective single-center study of 261 patients with a median follow-up of 1 year. Obes Surg. 2010; 20(3): 276-82.

8. Silecchia G, Boru C, Pecchia A, et al: Effectiveness of laparoscopic sleeve gastrectomy (first stage of biliopancreatic diversion with duodenal switch) on co-morbidities in super-obese highrisk patients. Obes Surg. 2006; 16(9): 1138-44.

9. Berende CA, de Zoete JP, Smulders JF, et al: Laparoscopic sleeve gastrectomy feasible for bariatric revision surgery. Obes Surg. 2012; 22(2): 330-4.

10. Melissas J, Koukouraki S, Askoxylakis J, et al: Sleeve gastrectomy: a restrictive procedure? Obes Surg. 2007; 17(1): 57-62.

11. Noah J, Smith A, Birch D, et al: The metabolic effects of laparoscopic sleeve gastrectomy: A review. J Minim Invasive Surg Sci. 2013; 2(3): 3-7.

12. Karamanakos SN, Vagenas K, Kalfarentzos F, et al: Weight loss, appetite suppression, and changes in fasting and postprandial ghrelin and peptide-YY levels after Roux-en-Y gastric bypass and sleeve gastrectomy: a prospective, double blind study. Ann Surg. 2008; 247(3): 401-7.

13. Sakran N, Goitein D, Raziel A, et al: Gastric leaks after sleeve gastrectomy: - multicenter experience with 2,834 patients. Surg Endosc. 2013; 27(1): 240-5. 
14. Frezza EE: Laparoscopic vertical sleeve gastrectomy for morbid obesity. The future procedure of choice? Surg Today. 2007; 37(4): 275-81.

15. Sarkhosh K, Birch DW, Sharma A, et al: Complications associated with laparoscopic sleeve gastrectomy for morbid obesity: A surgeon's guide. Can J Surg. 2013; 56(5): 34752.

16. Sajid MS, Khatri K, Singh K, et al: Use of stapleline reinforcement in laparoscopic gastric bypass surgery: a meta-analysis. Surg Endosc. 2011; 25(9): 2884-91.

17. Finks JF, Carlin A, Share D, et al: Effect of surgical techniques on clinical outcomes after laparoscopic gastric bypass-results from the Michigan Bariatric Surgery Collaborative. Surg Obes Relat Dis. 2011; 7(3): 284-9.

18. Dapri G, Cadiere GB, Himpens J: Reinforcing the staple line during laparoscopic sleeve gastrectomy: Prospective randomized clinical study comparing three different techniques. Obes Surg. 2010; 20(4): 462-7.

19. Albanopoulos K, Alevizos L, Flessas J, et al: Reinforcing the staple line during laparoscopic sleeve gastrectomy: Prospective randomized clinical study comparing two different techniques. Preliminary result. Obes Surg. 2012; 22(1): 42-6.

20. Himpens J, Dobbeleir J, Peeters G: Long-term results of laparoscopic sleeve gastrectomy for obesity. Ann Surg. 2010; 252(2): 319-24.

21. Osland E, Yunus RM, Khan S, Alodat T, Memon $B$, Memon MA: Postoperative early major and minor complications in laparoscopic vertical sleeve gastrectomy (LVSG) versus laparoscopic roux-en-Y gastric bypass (LRYGB) procedures: a meta-analysis and systematic review. Obes Surg. 2016; 26(10): 2273-84.

22. Gagner M, Hutchinson C, Rosenthal R: Fifth
International Consensus Conference: Current status of sleeve gastrectomy. Surg Obes Relat Dis. 2016; 12(4): 750-6.

23. Hany $M$, Ibrahim $M$ : Comparison between stable line reinforcement by barbed suture and non-reinforcement in sleeve gastrectomy: A randomized prospective controlled study. Obes Surg. 2018; 28: 2157-2164.

24. Gayrel X, Loureiro M, Skalli EM, Dutot C, Mercier G, Nocca D: Clinical and economic evaluation of absorbable staple line buttressing in sleeve gastrectomy in high-risk patients. Obes Surg. 2016; 26(8): 1710-1716.

25. Durmush EK, Ermerak G, Durmush D: Shortterm outcomes of sleeve gastrectomy for morbid obesity: Does staple line reinforcement matter? Obes Surg. 2014; 24(7): 1109-1116.

26. Romero RJ, Kosanovic R, Rabaza JR, et al: Robotic sleeve gastrectomy: Experience of 134 cases and comparison with a systematic review of the laparoscopic approach. Obes Surg. 2013; 23(11): 1743-52.

27. Abd Ellatif ME, Abbas A, El Nakeeb A, et al: Management options for twisted gastric tube after laparoscopic sleeve gastrectomy. Obes Surg. 2017; 27: 2404-9. OBES SURG.

28. Murcia $\mathrm{CH}$, Quintero PG, Rabaza J, Gonzalez A: Laparoscopic management of gastric torsion after sleeve gastrectomy. CRSLS.00143. 2014.

29. De Godoy EP, Coelho D: Gastric sleeve fixation strategy in laparoscopic vertical sleeve gastrectomy. Arq Bras Cir Dig. 2013;26 (Suppl 1): 79-82.

30. Gagner $M$, Kemmeter $P$ : Comparison of laparoscopic sleeve gastrectomy leak rates in five staple-line reinforcement options: A systematic review Surg Endosc. 2020; 34(1): 396-407. 